\title{
WHO-STATISTIK
}

\section{Teetrinker erkranken seltener an Diabetes}

— Leben Teetrinker gesünder? In einer Studie mit Teilnehmern aus 50 Nationen war Schwarzteekonsum invers mit der Häufigkeit von Diabetes verknüpft.

Das internationale Autorenteam beruft sich auf Daten des World Health Survey, einer von der WHO in Auftrag gegebenen jährlichen Gesundheitsbefragung, an der insgesamt 192 Nationen beteiligt sind. Das Augenmerk der Forscher lag auf dem Zusammenhang von Schwarzteekonsum mit

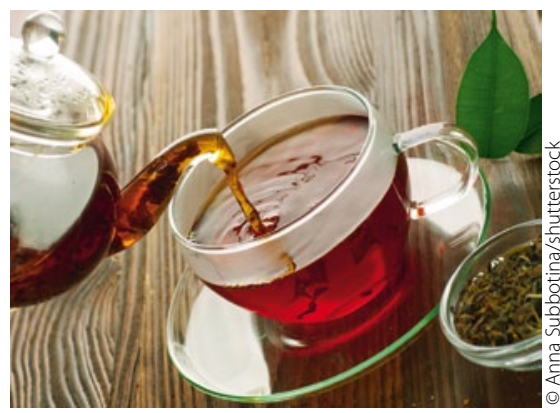

Teekultur - Schluck für Schluck gegen den Diabetes?
Atemwegserkrankungen, Infektionen, Krebs, kardiovaskulären Erkrankungen und Diabetes. Herausgegriffen wurden Daten aus 50 Nationen für das Jahr 2009. Demnach ergab sich allein für Diabetes eine klare inverse Korrelation, alle anderen erhobenen Gesundheitsrisiken schienen vom Teetrinken nicht beeinflusst. Die aktuell im British Medical Journal publizierten Daten bestätigen das Ergebnis einer ähnlichen Analyse aus Europa (InterAct Consortium 2012), die ebenfalls einen umgekehrten linearen Zusammenhang zwischen Teekonsum und Typ-2-Diabetes gezeigt hatte.

Aus Sicht der Autoren um Dr. Ariel Beresniak vom Data Mining International-Institut in Genf wäre nun mit einer randomisierten kontrollierten Studie zu klären, inwieweit Schwarztee wirklich in der Lage ist, den Blutzuckerstoffwechsel günstig zu beeinflussen und Diabetes zu verhindern. EO .

Beresniak A et al. BMJ Open 2012;2:e000648; doi:10.1136/bmjopen-2011-000648

\section{DÄNISCHE STUDIE}

\section{Senken Statine die Krebssterblichkeit?}

- Krebspatienten, die Statine einnehmen, haben eine im Schnitt 15\% niedrigere krankheitsspezifische Sterblichkeit als Tumorkranke, die keine Cholesterinsenker nehmen.

Mediziner der Universität Kopenhagen haben aus dem dänischen Melderegister die Daten von knapp 296000 Patienten abgeschöpft, die zwischen 1995 und 2007 eine Krebsdiagnose gestellt bekamen. Rund 19000 hatten schon davor regelmäßig Statine eingenommen, 277000 waren vor der Diagnose weder mit Statinen noch mit anderen cholesterinsenkenden Medikamenten behandelt worden.

Die Krebsmortalität der statinbehandelten Patienten war im Follow-up bis Ende 2009 um $15 \%$ geringer als die Malignomsterblichkeit der nicht mit Fettsenkern therapierten Tumorkranken. Die Tagesdosis der eingenommenen CSE-Hemmer schlug sich nicht in der Mortalitätsreduktion nieder. Für 13 Tumorentitäten war die Senkung der Sterblichkeit statistisch signifikant, am ausgeprägtesten für das Zervixkarzinom (minus $36 \%$ ). Die Mortalität von Lungenkrebspatienten sank um $13 \%$ und die von Kolonkrebskranken um 21\%. Bei Prostatakrebs wurde die Sterblichkeit um 19\% und bei Brustkrebs um 12\% reduziert.

Die dänischen Forscher halten ihre Ergebnisse für plausibel, weil Statine die Cholesterinsynthese hemmen. Unterbrechungen der entsprechenden Stoffwechselwege in malignen Zellen behindern das Wachstum und die Metastasierung von Krebs. Überdies haben Krebszellen einen hohen Bedarf an extrazellulärem Cholesterin. Prospektive Studien zu dem Thema stehen noch aus.

$\mathrm{RB}$ =

Nielsen SF et al. N Engl J Med 2012; 367: 1792-802; doi: 10.1056/NEJMoa1201735 